hep-th/yymmnnn

SCIPP-2009/01

\title{
Supersymmetry Breaking at Low Energies
}

\author{
Michael Dine \\ Santa Cruz Institute for Particle Physics and \\ Department of Physics, University of California, Santa Cruz CA 95064
}

\begin{abstract}
These lectures, given at the Cargese Summer school in 2008, provide an introduction to dynamical supersymmetry breaking and gauge mediation, with emphasis on the recent appreciation of the possible role of metastable supersymmetry breaking, and the evolving understanding of General Gauge Mediation. The underlying focus is on how supersymmetry might be realized at the Large Hadron Collider.
\end{abstract}




\section{Supersymmetry on the Eve of the LHC}

As we await collisions at the LHC, it seems a good time to assess various proposals for physics beyond the Standard Model. Certainly the possibility which has gained the most attention is that nature is approximately supersymmetric, with supersymmetry broken at the TeV scale. There are at least four reasons for this:

1. Supersymmetry can naturally account for the enormous hierarchy between the Planck scale and the weak scale.

2. With the assumption that all new thresholds lie at about $1 \mathrm{TeV}$, the gauge couplings unify reasonably well, at a scale of order $10^{16} \mathrm{GeV}$.

3. With the additional assumption of a conserved $R$ parity (the simplest hypothesis through which to forbid rapid proton decay), the theory automatically possesses a dark matter candidate, produced in abundance comparable to the observed dark matter density.

4. Supersymmetry arises rather naturally in many string constructions, and might plausible be broken by low energy, non-perturbative dynamics.

Each of these points, however, is open to serious challenge, and one is entitled to be indeed should be - skeptical that we are on the brink of the discovery of an extraordinary, previously unknown, symmetry of nature.

1. Current experimental constraints still require some fine tuning of Higgs parameters - as severe as $1 \%$ in many models. This is referred to as the "little hierarchy" problem.

2. Unification is not quite perfect, and depends on details of threshold effects at high and low scales. Unification itself, within, say our current understanding of string theory, is not automatic and it is unclear in what sense it is generic. Still, the success of the unification calculation strikes many physicists as remarkable.

3. Some degree of tuning is often required, given experimental constraints, to actually obtain the observed dark matter density.

4. As string theorists have explored the so-called string "landscape", questions have been raised as to just how generic low energy supersymmetry may be in string theory. They have also raised the worry that the hierarchy problem may ultimately have an anthropic explanation, similar to that which has been offered for the cosmological constant[1].

While theorists debate the significance of each of these points, there is little dispute that the various alternative proposals (technicolor, large or warped extra dimensions) have even more serious problems. In these lectures, we will adopt the optimistic viewpoint that these challenges may provide hints as to how supersymmetry is realized in nature. As we will review, the last few years have seen significant progress in understanding supersymmetric dynamics, and have opened up new possibilities for model building. So it is a good time to attempt to construct complete theories of lo energy supersymmetry and its breaking. 


\section{Challenges for Supersymmetry}

If the challenges to supersymmetry are clues to the underlying structure, it is important to understand them well. This is the focus of this section.

\subsection{The Little Hierarchy}

The little hierarchy may well indicate that theorists are on the wrong track. But if the supersymmetry hypothesis is correct, it suggests, we will see, a relatively low scale of supersymmetry breaking. The issues are nicely illustrated by ignoring gauge interactions, and just examining the top quark Yukawa couplings. From the top quark loop, one gets a quadratically divergent correction to the Higgs mass:

$$
\delta m_{H_{U}}^{2}=-6\left|y_{t}\right|^{2} \int^{\Lambda} \frac{d^{4} k}{(2 \pi)^{4}} \frac{1}{k^{2}-m_{t}^{2}},
$$

where $\Lambda$ is some cutoff. Without supersymmetry, This result is proportional to some cutoff scale squared. This is the usual statement of the hierarchy problem; without supersymmetry, masses of scalars are expected, by dimensional analysis, to be of order $\Lambda^{2}$, where $\Lambda$ is some scale where the effective lagrangian which describes their interactions breaks down (e.g. due to compositeness, some modification of the structure of space-time, or something equally dramatic).

With supersymmetry, the diagrams with the stops give a contribution which cancels the leading divergence:

$$
\delta m_{H_{U}}^{2(2)}=6\left|y_{t}\right|^{2} \int \frac{d^{4} k}{(2 \pi)^{4}} \frac{1}{k^{2}-\tilde{m}_{t}^{2}} .
$$

There is still a subleading logarithmic divergence, which, for $\tilde{m}_{t}^{2} \gg m_{t}^{2}$ yields:

$$
\delta m_{H_{U}}^{2} \approx-6 \tilde{m}_{t}^{2} \frac{y_{t}^{2}}{16 \pi^{2}} \ln \left(\frac{\Lambda^{2}}{\tilde{m}_{t}^{2}}\right)
$$

where $\Lambda$ is an ultraviolet cutoff, and we have neglected $m_{t}^{2}$ relative to $\tilde{m}_{t}^{2}$. The quadratic divergence has been replaced by a logarithmic one; the severe hierarchy problem which exists in the absence of supersymmetry is solved, provided that the scale of supersymmetry breaking - the masses of the various superpartners - is not much greater than the weak scale.

Without supersymmetry, assuming the cutoff is large, say of order $M_{\text {gut }}=2 \times 10^{16} \mathrm{GeV}$, the correction to the Higgs mass is enormous; in order that the mass be of order, say, $100 \mathrm{GeV}$, one needs to introduce a bare mass parameter of order $10^{30} \mathrm{GeV}^{2}$, which cancels against the radiative correction up to one part in $10^{26}$. So supersymmetry yields an enormous improvement. But there is still a difficulty. As we will explain shortly, within simple supersymmetric models, the physical Higgs mass cannot be much larger $m_{Z}$. Let's examine eqn. 3 in view of this 
constraint. Limits on the stop mass are in the $350 \mathrm{GeV}$ range. If we take the cutoff, $\Lambda$, to be of order $M_{0}$, we then obtain a correction

$$
\frac{\Delta m_{H_{U}}^{2}}{m_{Z}^{2}} \approx 40
$$

i.e. a tuning of order $2 \%$. But the situation may be even worse than this; in many models, there are arguments that the stop mass should be significantly larger, $800 \mathrm{GeV}$ or more. In that case, the fine tuning is more like a part in 200 !

If we are to ameliorate this, we need to suppress both the logarithm and the stop mass. For example, suppose that the cutoff is $10 \mathrm{TeV}$ (we will motivate this choice shortly), and that

the stop mass is close to the experimental limit. Then $\Delta m_{H_{U}}^{2} \sim 4 m_{Z}^{2}$, arguably not fine tuned at all.

What, physically, is the cutoff scale $\Lambda$ ? In so-called supergravity models, as they are not renormalizable, it is naturally thought of as of order the Planck scale (perhaps the string scale, if we imagine the theory is embedded in string theory). As we will see, however, the cutoff can be much lower. Indeed, in general, the scale is related to the scale associated with the messengers of supersymmetry breaking.

These considerations provide one motivation for thinking about supersymmetry breaking at low scales. Indeed, they suggest that supersymmetry should be broken at an underlying scale close to the 100's of GeV scale of squarks and sleptons. A widely studied framework which might achieve this is known as gauge mediation[2].

\subsection{The Proliferation of Parameters}

My experimental colleagues often express frustration with theorists: they have already presented us with 20 or so parameters to explain, and rather than do so, we seem committed to introducing more. At a minimum, supersymmetry introduces 105 new parameters. Counting of these parameters in the simplest generalization of the Standard Model, the Minimal Supersymmetric Standard Model (MSSM) is very simple, and worth doing. I'll define the MSSM by its particle content: it is a theory in which each fermion of the standard model is replaced by a chiral superfield, each gauge boson by a vector field, and the higgs bosons by a pair of chiral fields, $H_{U}, H_{D}$. The gauge quantum numbers of the quark and lepton superfields are just those of the Standard Model; the Higgs superfields, $H_{U}$ and $H_{D}$, are doublets, with hypercharge \pm 1 . (See, for example, [5], chapter 11, for an introduction). It is necessary to have at least two Higgs doublets in order to avoid anomalies (perturbative and non-perturbative). In order to explain the observed features of quark and lepton masses, it is natural to suppose that the superpotential contains a generalization of the Standard Model Yukawa couplings:

$$
W_{y}=y_{U} H_{U} Q \bar{U}+y_{D} H_{D} Q \bar{D}+y_{L} H_{D} \bar{E} .
$$

Here $y_{U}$ and $y_{D}$ are $3 \times 3$ matrices in the space of flavors. 
But additional couplings are permitted, and here we encounter some troubling features. First, for the Higgs fields, we can, and will, add a " $\mu$ " term,

$$
W_{\mu}=\mu H_{U} H_{D}
$$

If $\mu$ is too large, the Higgs fields are too massive to play a role in electroweak symmetry breaking; if it is too small, one can't obtain an acceptable chargino and neutralino spectrum. In supersymmetric theories, as I'll explain, there is at most logarithmic renormalization of $\mu$, but still, dimensional analysis would suggest that it should be of order GUT or Planck scale. One might imagine it's small value arises due to an underlying symmetry; string theory has also provided a variety of mechanisms which might provide an explanation. Still, the fact that this is not automatic is, at first sight, disappointing.

Second, there are a class of couplings which violate lepton and/or baryon number:

$$
W_{b / l-v i o l a t i n g}=\Gamma_{1} \bar{U} \bar{D} \bar{D}+\Gamma_{2} Q L \bar{D}+\Gamma_{3} L L \bar{E} .
$$

The couplings $\Gamma$ are dimensionless, and thus might be expected to be of order one - and hence catastrophic. The simplest approach to this problem is to banish these couplings entirely: impose a discrete symmetry, called " $R$ parity", under which the partners of ordinary fields (squarks, sleptons, gauginos, higgsinos) are odd, and ordinary fields are even. Again, it is somewhat disappointing that one needs to impose yet another requirement on these theories, but at least symmetries are natural, and indeed symmetries of this type often arise in string theory. Moreover, imposing this symmetry brings a bonus: the lightest of the new supersymmetric particles, the LSP, is stable, and potentially a candidate for the dark matter. It should be noted, as well, that other discrete symmetries which treat fermions and bosons differently $(R$ symmetries) can achieve this result, with other potentially interesting consequences (e.g. such a symmetry might explain the smallness of $\mu$, or the very strong limits on the proton lifetime).

With these caveats, we are ready to count parameters. So far, we have roughly the same number of parameters as the Standard Model. But supersymmetry is clearly broken in nature, and the masses of squarks, sleptons and gauginos are subject to severe experimental constraints. So we add to the lagrangian a set of soft supersymmetry breaking terms. The allowed terms (those which don't reintroduce power law divergences are easily classified[3, 4]; see [5], pp. 162-163 for a simple derivation), and include:

1. Soft mass terms for squarks, sleptons, and Higgs fields:

$$
\begin{gathered}
\mathcal{L}_{\text {scalars }}=Q^{*} m_{Q}^{2} Q+\bar{U}^{*} m_{U}^{2} \bar{U}+\bar{D}^{*} m_{D}^{2} \bar{D} \\
+L^{*} m_{L}^{2} L+\bar{E}^{*} m_{E} \bar{E} \\
+m_{H_{U}}^{2}\left|H_{U}\right|^{2}+m_{H_{U}}^{2}\left|H_{U}\right|^{2}+B_{\mu} H_{U} H_{D}+\text { c.c. }
\end{gathered}
$$

$m_{Q}^{2}, m_{U}^{2}$, etc., are matrices in the space of flavors. The first five matrices are $3 \times 3$ Hermitian matrices (45 parameters); the Higgs mass terms add an additional 4, for a total of 49 parameters. 
2. Cubic couplings of the scalars:

$$
\begin{gathered}
\mathcal{L}_{\mathcal{A}}=H_{U} Q A_{U} \bar{U}+H_{D} Q A_{D} \bar{D} \\
+H_{D} L A_{E} \bar{E}+\text { c.c. }
\end{gathered}
$$

Here the matrices $A_{U}, A_{D}, A_{E}$ are complex matrices, so we have an additional 54 parameters.

3. Mass terms for the $\mathrm{U}(1)(b), S U(2)(w)$, and $S U(3)(\lambda)$ gauginos:

$$
m_{1} b b+m_{2} w w+m_{3} \lambda \lambda
$$

These are three complex quantities, making six additional parameters.

So we would seem to have an additional 109 parameters. However, the supersymmetric part of the MSSM lagrangian has symmetries which are broken by the general soft breaking terms (including $\mu$ among the soft breakings):

1. Two of three separate lepton numbers

2. A "Peccei-Quinn" symmetry, under which $H_{U}$ and $H_{D}$ rotate by the same phase, and the quarks and leptons transform suitably.

3. A continuous " $R$ " symmetry, which we will explain in more detail below.

Redefining fields using these four transformations reduces the number of parameters to 105.

\section{$2.3 \quad$ Aside on R Symmetries}

In the previous section, we mentioned the $R$ symmetry of the MSSM which exists in the absence of soft breakings. In general, an $R$ symmetry is a symmetry under which the supercharges rotate (equivalently, for which the generator does not commute with the supercharges). This is possible since the Hamiltonian is quadratic in the charges. Under a continuous $R$ symmetry, if we define the Grassmann coordinates, $\theta$, to transform with phase $e^{i \alpha}$, the supercharges $Q_{\alpha}$ transform with the opposite phase, and the superpotential transforms with phase $e^{2 i \alpha}$. A model respects a continuous $R$ symmetry if it is possible to assign charges to all fields so that the Kahler potential is invariant and the superpotential transforms with $R$ charge 2. Gauginos transform with charge 1; the fermions in chiral multiplets transform with one unit less charge than the scalars.

In the case of the supersymmetric couplings of the MSSM, including the $\mu$ term, one can assign: $H_{U}, H_{D} R$ charge 1 and all of the quark and lepton superfields $R$ charge $1 / 2$. (Other assignments differ by combinations of baryon number and other symmetries).

In general, we don't expect global continuous symmetries in fundamental theories; when

they arise, as in the case of baryon number in the Standard Model, they should be accidental 
consequences of renormalizability and other symmetries. On the other hand, discrete $\mathrm{R}$ symmetries are plausible; they are quite common in string theory, for example (arising, for example, as unbroken subgroups of higher dimensional rotation groups upon compactification). At the level of renormalizable couplings, such symmetries could well lead to continuous symmetries. We have now seen that this occurs in the MSSM, once one supposes an $R$ parity (perhaps the simplest of discrete $R$ symmetries).

\subsection{Constraints on the Soft Parameters}

Ignoring the Higgs Yukawa couplings, the fields of the Standard Model respect a large set of flavor symmetries. Since the quark masses (apart from the top quark) are small compared to the masses of the $W$ and $Z$ bosons, these flavor symmetries play an important role in the phenomena of weak interactions. A priori, there is no reason for the squark and slepton masses to respect these symmetries. But if these masses are of order the weak scale, and are simply random numbers, the effects on flavor changing processes can be dramatic.

The most stringent constraints come from study of the neutral kaon system. One of the early triumphs of the Standard Model was its explanation of the small rates of "neutral current" processes in this system $\left(K^{0} \leftrightarrow \bar{K}^{0}, K^{0} \rightarrow \mu \bar{\mu}\right.$, etc.). These effects are suppressed not only by $G_{F}$ and a loop factor, but, due to the approximate flavor symmetries mentioned above, an additional factor of $m_{c}^{2} / M_{W}^{2}$, i.e. nearly $10^{-4}$. Supersymmetry introduces many new contributions to these processes. Exchange of gluinos and squarks, for example, gives a contribution to $K-\bar{K}$ mixing which is suppressed, for general values of the masses, only by $\frac{\alpha_{s}}{4 \pi} \frac{1}{m_{s u s y}^{2}}$, not nearly enough.

Exercise: Estimate the gluino box contribution to $K \bar{K}$ mixing by comparing to the ordinary weak interactions. Unless you are careful about chirality, you will underestimate the contribution by nearly an order of magnitude!

These problems are further exacerbated when one allows for $\mathrm{CP}$ violating phases in the soft terms. If these phases are simply numbers of order one, the constraints become tighter by an order of magnitude.

One can satisfy all of these constraints at once if one assumes that the squark and slepton masses are all degenerate at some energy scale. More precisely, one assumes that each of the matrices $m_{Q}, m_{\bar{U}}$, etc., are proportional to the unit matrix, while the matrices $A_{U}, A_{D}$, etc., are proportional to the Yukawa couplings. This automatically suppresses flavor changing processes in the light meson systems; any violation of flavor is proportional to small Yukawa couplings and mixing angles. Masiero[6] carefully reviews the constraints on these processes, and translates them into constraints on the degree of degeneracy of squark and slepton masses.

So if supersymmetry breaking is at a low scale, the 105 or more new parameters associated with supersymmetry are highly constrained. Most analyses of supersymmetry phenomenology and model building simply take some level of degeneracy as a given. For example, most ex- 
perimental studies are based on the assumption that the spectrum can be described by three parameters, $m_{0}^{2}, m_{1 / 2}, A$, $\mu$, the first being a common value of the scalar masses at some high scale (e.g. the unification scale), the second the common value of the gaugino masses, the third a common $A$ term, and the fourth the $\mu$ term in the superpotential we have discussed earlier.

One can ask how natural is this assumption? The set of parameters described above are often called "minimal supergravity", the idea being that gravity is universal, and supergravity should be as well. But, as we will see in section 3, a supergravity theory with the field content at low energies of the MSSM has precisely the number of independent parameters we have counted above, and there is no symmetry which forces any degree of degeneracy. Still, except for the top Yukawa, all of the Yukawa couplings are small, and it seems plausible that there might be some approximate flavor symmetry which could account for degeneracy. Microscopic models with such symmetries have been considered by many authors, and dynamical explanations have been suggested as well for accidental flavor symmetries (particularly involving large extra dimensions). But probably the simplest implementation is provided by models of "gauge mediation" [2]. Gauge mediated models can be defined as field theory models where, in the limit that the gauge couplings tend to zero, there is no coupling between the dynamics which breaks supersymmetry ("hidden sector") and the fields of ordinary physics[11]. Given the assumption that there is a good effective field theory description at a scale below that which determines the quark and lepton Yukawa couplings, these couplings can be treated as perturbations. This definition, as we will discuss, requires modification in order to account for the $\mu$ term, and any modification risks reintroducing flavor violations. But this simple success in providing a generic framework for the absence of flavor changing neutral currents is one of the principle reasons for interest in gauge mediation.

That said, flavor violation in light meson systems is not the only constraint which low energy physics places on the soft breaking parameters. There are at least two additional striking facts which any model must account for. First are the constraints on electric dipole moments of quarks (the neutron) and the electron. For example, for quark electric dipole moments one might expect

$$
d_{q} \approx \frac{\alpha_{s}}{4 \pi} \frac{m_{q}}{m_{\lambda}^{2}} \sin (\delta)
$$

where the gluino mass here represents some average of masses appearing in the one loop contribution, and $\delta$ is a CP-violating phase. For the $d$ quark, and assuming a large value, say 1 $\mathrm{TeV}$ for the gluino mass, one obtains about $2 \times 10^{-24} \sin \delta$ e-cm, which is more than an order of magnitude above the experimental limit; things are significantly worse if the gluino is lighter. So one needs to somehow explain the suppression of the phase $\delta$. The second constraint is the rate for the inclusive process $b \rightarrow s+\gamma$. Current experimental data and Standard Model calculations leave a small amount of room for possible new physics contributions. However, the charged Higgs contribution in the MSSM tends to be too large, unless the Higgs is rather heavy[7]. 


\subsection{The Higgs Mass}

Since the conclusion of the LEP II program, there is a very severe constraint on models of supersymmetry: the Higgs mass itself. In the MSSM, as we have remarked, it is easy to show that there is a strict limit on the Higgs mass at the classical level: $m_{H}<M_{Z}$. This result is not surprising. In the Standard Model, the Higgs mass is proportional to the Higgs quartic coupling; in the MSSM, the Higgs quartic couplings arise entirely from gauge interactions. Because the top Yukawa coupling is so large (of order one), however, there is the potential for large radiative corrections. One loop corrections generate a substantial supersymmetry-violating Higgs quartic coupling. It is easy to isolate a contribution which diverges logarithmically as the stop mass tends to infinity. In the large $\tan \beta$ limit, and assuming that the left and right-handed stop quark masses are identical, there is a correction to the $\left|H_{U}\right|^{4}$ coupling:

$$
\delta \lambda=\frac{3 y_{t}^{4}}{16 \pi^{2}} \ln \left(\tilde{m}_{t}^{2} / m_{t}^{2}\right) .
$$

Exercise: Verify eqn. 12.

Plugging this into the potential, and working out the corresponding physical Higgs mass, one finds that requiring a Higgs mass of at least $116 \mathrm{GeV}$ requires that $\tilde{m}_{t}>800 \mathrm{GeV}$. From the point of view of fine tuning we described earlier, this is troubling. More complete computations are readily found in the literature. If one allows significant mixing between the left and righthanded stops, the situation is not necessarily as severe (for a recent discussion, see [8]).

Alternatively, additional fields and interactions can alter the situation. The simplest possibility, known as the NMSSM (for Next to Minimal Supersymmetric Supersymmetric Standard Model) contains an additional singlet, $S$, with coupling $\lambda_{S} S H_{U} H_{D}$. This provides an additional quartic Higgs couplings, and can enlarge (slightly) the allowed range of Higgs masses.

More generally, if there is some new physics at a scale, $M$, slightly above the scale of the Higgs fields, one can integrate out this physics and represent its effects in a local effective lagrangian[9]. Corrections to quartic couplings will be down by powers of $M$, so the lowest dimension operators will be most important. There is a unique operator of dimension five which generates a quartic coupling:

$$
\mathcal{O}=\frac{1}{M} \int d^{2} \theta H_{U} H_{D} H_{U} H_{D}
$$

Solving for the auxiliary fields, gives a quartic coupling, of order $\mu / M$. The NMSSM can be understood in this way, in the case that the mass of the singlet is larger than $\mu$. These terms are most important when $H_{U} \sim H_{D}$ (the region of "small $\tan \beta$ "). If $H_{U} \gg H_{D}$, dimension six operators are most important. In either case, such effects can yield a Higgs of mass greater than the experimental bound, but masses much larger than that seem hard to achieve. 


\section{Supergravity Models}

Despite our somewhat disparaging remarks about supergravity models and the problem of flavor, these theories provide a simple conceptual framework in which to consider supersymmetry breaking and its mediation to low energy physics. They are a natural setting, as well, to think about the possible phenomenologies which might emerge from string theory. The bosonic part of the general supergravity lagrangian, including terms with at most two derivatives, can be described rather simply. The lagrangian is specified by three functions: the Kahler potential, superpotential and gauge coupling function, $K\left(\Phi_{i}, \Phi_{i}^{*}\right), W\left(\Phi_{i}\right)$, and $f_{a}\left(\Phi_{i}\right)$. The Kahler potential is a general function of the fields, while the superpotential and gauge coupling function (the latter is really several functions) are holomorphic. The scalar potential is given by:

$$
\begin{aligned}
V= & e^{K / M_{p}^{2}}\left[\left(\frac{\partial W}{\partial \Phi_{i}}+\frac{1}{M_{p}^{2}} \frac{\partial K}{\partial \Phi_{i}} W\right) g^{i \bar{j}}\right. \\
& \left.\left(\frac{\partial W}{\partial \Phi_{\bar{j}}^{*}}+\frac{1}{M_{p}^{2}} \frac{\partial K}{\partial \Phi_{\bar{j}}^{*}} W^{*}\right)-3|W|^{2}\right]
\end{aligned}
$$

to which must be added the terms proportional to $\left(D^{a}\right)^{2}$ from the gauge couplings. The condition for unbroken susy is that the "Kahler derivative" of $W$ with respect to all fields should vanish:

$$
D_{i} W=0 \quad D_{i} W=\frac{\partial W}{\partial \phi_{i}}+\frac{\partial K}{\partial \Phi_{i}} W
$$

In addition, the auxiliary fields for all of the gauge groups must vanish.

Now to make a model of low energy physics, we assume that

1. Supersymmetry is broken in a hidden sector, involving some fields $X_{\alpha}$; the visible sector fields will be denoted $\phi_{i}$.

2. The Kahler potential has a very simple form:

$$
K=\sum \phi_{i}^{*} \phi_{i}+X_{\alpha}^{*} X_{\alpha}
$$

This potential has a $U(N)$ symmetry, where $N$ is the number of fields; this symmetry will suppress flavor changing neutral currents. The scalar mass terms are universal, and the soft breaking cubic terms are proportional to the fermion Yukawa couplings.

3. The superpotential is a sum of hidden sector and visible sector fields:

$$
W=f\left(\phi_{i}\right)+g\left(X_{\alpha}\right) .
$$

4. $g\left(X_{\alpha}\right)$ is assumed to break supersymmetry, $D_{\alpha} W \neq 0$.

5. Gaugino masses arise from a universal gauge coupling function of the form

$$
f(X)=f(0)+c_{\alpha} \frac{X_{\alpha}}{M}
$$


6. A $\mu$ term can arise from a term in the Kahler potential such as $X^{\dagger} H_{U} H_{D}$. Such couplings are often generated by integrating out heavy fields.

Before listing the problems of these theories, some of their features should be noted. The mass of the gravitino, assuming that the space-time is flat, is given by

$$
m_{3 / 2}=\left\langle e^{K / 2} W_{0}\right\rangle .
$$

Scalar masses arise from terms in the potential such as

$$
\left|\frac{\partial W}{\partial \phi_{i}}+\phi_{i}^{*} W\right|^{2} \sim\left|\phi_{i}\right|^{2} m_{3 / 2}^{2}
$$

$A$ terms arise from a variety of sources, such as

$$
\left(\frac{\partial W}{\partial X}\right)^{*} \frac{\partial K}{\partial X} W
$$

where one takes the term in $W$ which is cubic in fields $\left(H_{U} Q \bar{U}\right.$, etc), and replacing $X$ by its vev.

The assumptions underlying the model, however, are quite strong. As we have remarked, the $U(N)$ symmetry assumed in the Kahler potential cannot reasonably be expected to hold in a general situation, even approximately. Terms like $X X^{\dagger} Q Q^{\dagger}$, with various flavor indices, will generate new, non-universal terms in the mass matrices and $A$ terms. In string theory, various scenarios have been put forth which might give rise to accidental symmetries of the type required, but it is not at all clear how plausible these are.

\section{Minimal Gauge Mediation}

We have already described the main premiss underlying gauge mediation: in the limit that the gauge couplings vanish, the hidden and visible sectors decouple. A simple model illustrates the basic idea. Suppose we have a chiral field, $X$, with

$$
\langle X\rangle=x+\theta^{2} F .
$$

Suppose also that $X$ is coupled to a vector-like set of fields, transforming as a single 5 and $\overline{5}$ of $S U(5)$ :

$$
W=X\left(\lambda_{\ell} \bar{\ell} \ell+\lambda_{q} \bar{q} q\right)
$$

For $F<X, \ell, \bar{\ell}, q, \bar{q}$ are massive, with supersymmetry breaking splittings of order $F$. The fermion masses are given by:

$$
m_{q}=\lambda_{q} x \quad m_{\ell}=\lambda_{\ell} x
$$

while the scalar splittings are

$$
\Delta m_{q}^{2}=\lambda_{q} F \quad \Delta m_{\ell}^{2}=\lambda_{\ell} F
$$


In such a model, masses for gauginos are generated at one loop; for scalars at two loops. The gaugino mass computation is quite simple. Even the two loop scalar masses turn out to be rather easy, as one is working at zero momentum. The latter calculation can be done quite efficiently using supergraph techniques; an elegant alternative uses background field arguments[10]. The result for the gaugino masses is:

$$
m_{\lambda_{i}}=\frac{\alpha_{i}}{\pi} \Lambda
$$

while for the squark and slepton masses it is:

$$
\begin{gathered}
\widetilde{m}^{2}=2 \Lambda^{2}\left[C_{3}\left(\frac{\alpha_{3}}{4 \pi}\right)^{2}+C_{2}\left(\frac{\alpha_{2}}{4 \pi}\right)^{2}\right. \\
\left.+\frac{5}{3}\left(\frac{Y}{2}\right)^{2}\left(\frac{\alpha_{1}}{4 \pi}\right)^{2}\right],
\end{gathered}
$$

where $\Lambda=F_{x} / x . C_{3}=4 / 3$ for color triplets and zero for singlets, $C_{2}=3 / 4$ for weak doublets and zero for singlets.

This spectrum has a number of notable features.

1. One parameter describes the masses of the three gauginos and the squarks and sleptons

2. Flavor-changing neutral currents are automatically suppressed; each of the matrices $m_{Q}^{2}$, etc., is automatically proportional to the unit matrix; the $A$ terms are highly suppressed (they receive no one contributions before three loop order).

3. $\mathrm{CP}$ conservation is automatic

4. This model cannot generate a $\mu$ term; the term is protected by symmetries. Some further structure is necessary.

\subsection{Messenger Parity and $D$ terms}

The model of eqn. 23 neatly avoids a potential pitfall of this sort of construction. There are, in fact, individual one loop graphs which contribute to squark and slepton masses, corresponding to the possibility of an expectation value for the auxiliary field of the hypercharge multiplet, $\langle D\rangle$. $\langle D\rangle$ receives contributions from messenger fields, and can be non-vanishing because supersymmetry is broken. However, this model possesses an accidental, approximate symmetry under which

$$
q \leftrightarrow \bar{q} \quad \ell \leftrightarrow \bar{\ell} \quad V_{Y} \rightarrow-V_{Y}
$$

This symmetry is broken by the interactions of the MSSM, but this will only be visible at high loop order. It is easy to check the cancelation of the corresponding diagrams explicitly. 


\subsection{Minimal Gauge Mediation and the Little Hierarchy Problem}

In considering the little hierarchy, the first question to ask is "what is the cutoff $\Lambda$ in eqn. 3 . In renormalizable theories like those considered here, there cannot actually be a divergence. The one loop correction to the Higgs mass is really a three loop graph, in which the two loop subgraph responsible for the stop mass has been shrunk to a point. This description breaks down at the scale $M$ (the scale of the messenger masses). So, in MGM, $M$ is the cutoff. $M$,

can in principle be as small as $M \sim \frac{4 \pi}{\alpha_{s}}($ few hundred $\mathrm{GeV}) \sim$ few tens of $\mathrm{TeV}$. So the $\log$ need not be terribly large.

On the other hand, given the experimental constraints on the susy spectrum, the stop mass appearing in the loop is necessarily quite large in MGM. In particular, the experimental limits on the mass of the lightest charged slepton are of order $100 \mathrm{GeV}$ (the precise limit depends on the allowed decay channels). Satisfying this constraint implies, from the formulae for the scalar masses, that the squark masses are quite large, of order $800 \mathrm{GeV}$. So one has a fine tuning, at best, at the few per cent level.

If one could compress the spectrum, one could ameliorate the little hierarchy. In other words, if one had a spectrum in which squark and slepton masses were not so different, one could improve the situation significantly. Current experimental limits on the squark masses are $300-400 \mathrm{GeV}$, so fine tuning of less than a part in five may be possible. We will see shortly that such a compression of the spectrum can occur in more general models of messengers.

\section{General Gauge Mediation}

Much work has been devoted to understanding the properties of this simple model, but it is natural to ask: just how general are these features? It turns out that they are peculiar to our assumption of a single set of messengers and just one singlet responsible for supersymmetry breaking and R symmetry breaking. Meade, Seiberg and Shih[11] have formulated the problem of gauge mediation in a general way, and dubbed this formulation General Gauge Mediation (GGM). They study the problem in terms of correlation functions of (gauge) supercurrents. Analyzing the restrictions imposed by Lorentz invariance and supersymmetry on these correlation functions, they find that the general gauge-mediated spectrum is described by three complex parameters and three real parameters.

While we won't review the analysis of [11] in detail, it is easy to see, in simple weakly coupled models, how one can obtain a larger set of parameters. Take, for example, a model, as above, with messengers $q, \bar{q}, \ell, \bar{\ell}$, but replace the one singlet of the earlier model with a set of singlets, $X_{i}$. For the superpotential, take:

$$
W=\lambda_{i}^{q} X_{i} \bar{q} q+\lambda_{i}^{\ell} X_{i} \bar{\ell} \ell .
$$

Now, unlike the case of minimal gauge mediation, the ratio of the splittings in the multiplets to the average (i.e. fermion) masses is not the same for $q, \bar{q}$ and $\ell, \bar{\ell}$. For the fermion masses:

$$
m_{q}=\sum \lambda_{i}^{q} x_{i} \quad m_{\ell}=\sum \lambda_{i}^{\ell} x_{i}
$$


while the scalar splittings are

$$
\Delta m_{q}^{2}=\sum \lambda_{i}^{q} F_{i} \quad \Delta m_{\ell}^{2}=\sum \lambda_{i}^{\ell} F_{i} .
$$

In the case of MGM, the one loop contributions for fields carrying color were proportional to $\Delta m_{q}^{2} / m_{q}^{2}$, while those contributing to $\ell$ were proportional to $\Delta m_{\ell}^{2} / m_{\ell}^{2}$. One now finds, simply generalizing the previous computation, for the masses of the gauginos:

$$
\begin{array}{r}
m_{\lambda}=\frac{\alpha_{3}}{4 \pi} \Lambda_{q} \quad m_{w}=\frac{\alpha_{2}}{4 \pi} \Lambda_{\ell} \\
m_{b}=\frac{\alpha_{1}}{4 \pi}\left[2 / 3 \Lambda_{q}+\Lambda_{\ell}\right] .
\end{array}
$$

where

$$
\Lambda_{q}=\frac{\lambda_{q}^{i} F_{i}}{\lambda_{q}^{j} x_{j}} \quad \Lambda_{\ell}=\frac{\lambda_{\ell}^{i} F_{i}}{\lambda_{\ell}^{j} x_{j}}
$$

( $i$ and $j$ summed). Similarly, for the squark and slepton masses we have:

$$
\begin{array}{r}
\tilde{m}^{2}=2\left[C_{3}\left(\frac{\alpha_{3}}{4 \pi}\right)^{2} \Lambda_{q}^{2}+C_{2}\left(\frac{\alpha_{2}}{4 \pi}\right)^{2} \Lambda_{\ell}^{2}\right. \\
\left.+\left(\frac{Y}{2}\right)^{2}\left(\frac{\alpha_{1}}{4 \pi}\right)^{2}\left(\frac{2}{3} \Lambda_{q}^{2}+\Lambda_{\ell}^{2}\right)\right]
\end{array}
$$

At this point, it is easy to understand the parameter counting of Meade et al. We can write the general gauge-mediated spectrum in terms of three independent complex masses for the gauginos, and parameterize the general sfermion mass matrix as:

$$
\begin{gathered}
\widetilde{m}^{2}=2\left[C_{3}\left(\frac{\alpha_{3}}{4 \pi}\right)^{2} \Lambda_{q c d}^{2}+C_{2}\left(\frac{\alpha_{2}}{4 \pi}\right)^{2} \Lambda_{w}^{2}\right. \\
\left.+\left(\frac{Y}{2}\right)^{2}\left(\frac{\alpha_{1}}{4 \pi}\right)^{2} \Lambda_{b}^{2}\right],
\end{gathered}
$$

In the present case, there are two relations among these masses, which can be expressed as sum rules. But more generally, we have three independent complex parameters, the gaugino masses, and three additional real parameters.

Models with additional fields permit independent values for all of the parameters of GGM. In constructing examples, we will insist that the messengers fill complete multiplets of $S U(5)$, so as to preserve unification (one can legitimately ask why nature would be so concerned with achieving unification). For example, suppose one has a 10 and $\overline{1} 0$ of messengers, and multiple singlets. The messengers can be denoted as $Q, \bar{Q}, U, \bar{U}$, and $E, \bar{E}$. One now has three independent parameters, which, by analogy to our previous example, can be denoted as $\Lambda_{Q}, \Lambda_{U}, \Lambda_{E}$. The minimal, weak coupling theory which yields the full set of parameters of GGM consists of a 10 and $\overline{1} 0$, and two $5, \overline{5}$ pairs. In this case, however, if the scale of supersymmetry breaking is low, the gauge couplings tend to get strong well below the unification scale. In addition, there is not an automatic messenger parity, so it is necessary to require additional structure in order to suppress the Fayet-Iliopoulos term for hypercharge. Finally, these models don't actually cover the full parameter space (though they have the maximal number of parameters); a strategy for doing this is described in [13]. 


\section{The Higgs Sector in Gauge Mediation}

So far, we have adopted the definition of gauge mediation of [11], in which, in the limit that the gauge couplings all tend to zero, there is no supersymmetry breaking in the MSSM sector (for our discussion, we will again treat $\mu$ as a supersymmetry breaking parameter). But such a theory cannot be phenomenologically realistic. For example, there is necessarily a massless fermion from the Higgs sector, which is not compatible with measurements of the $Z$ width and other constraints, a relatively light chargino incompatible with experimental bounds, and a very light pseudoscalar. The latter arises because the theory, in this limit, has an exact symmetry broken only by anomalies, under which the Higgs fields transform by the same phase. The Higgs expectation values break this Peccei-Quinn symmetry, giving rise to a pseudogoldstone boson.

In [11], this problem was phrased in terms of operators which would couple Higgs to the messenger sector, breaking this additional symmetry. Ref. [12] provides a systematic analysis of this problem. If we write renormalizable (dimension three or four) couplings, then there are two types of couplings between the Higgs fields and messengers: couplings linear in the Higgs, and those quadratic. Linear couplings have to involve operators in the messenger sector with weak isospin $1 / 2$; quadratic couplings can involve singlet or triplet operators. The problem can, again, be organized in terms of correlation functions in the messenger sector. However, some of the issues can be illustrated by considering a simple model of elementary messengers coupled to the Higgs:

$$
W=\lambda_{u} H_{u} \ell X+\lambda_{d} H_{d} \ell X
$$

Here the $X$ field is the same as in eqn 23. If $\lambda_{u}$ and $\lambda_{d}$ are small, we can integrate out the messenger fields, already at tree level obtaining a $\mu$ and $B_{\mu}$ term:

$$
\mu \sim \lambda_{u} \lambda_{d} x \quad B_{\mu}=\lambda_{u} \lambda_{d} \frac{F}{M} .
$$

Now there are several issues. If we want $\mu$ and $B_{\mu}$ to be of order the weak scale, $\lambda_{u}$ and $\lambda_{d}$ should be small. From the expression for $\mu$, we need $\lambda^{2}$ of order a loop factor. But this means $\mu^{2}$ is two loop order, while $B_{\mu}$ is one loop order. This problematic hierarchy is typical of attempts to understand $\mu$ in gauge mediation. One out, noted in [12], is to add additional fields, with a symmetry which suppresses $B_{\mu}$. These models also have a potential problem with the $D$ term for hypercharge, but if $\lambda^{2}$ is of order a loop factor, then $\left\langle D_{Y}\right\rangle$ is of two loop order, which is small enough.

A number of alternative mechanisms to generate $\mu$ and $B_{\mu}$ have been proposed. One class of models, which generate the correct hierarchy naturally, involves a coupling of a singlet, $S$, to $H_{U} H_{D}$. The singlet also couples to messengers, in such a way that at two loop order one generates an expectation value for $S$, leading, in turn, to a $\mu$ and $B_{\mu}$ term, with $\mu^{2} \sim B_{\mu}[14]$.

The analysis of [12] allows a systematic classification of these possibilities, and construction of numerous models. One can debate whether these complications make gauge mediation more or less plausible. 


\section{$7 \quad$ Microscopic Models of Supersymmetry Breaking}

Simply introducing soft breaking parameters has two limitations:

1. The theory is not complete in the ultraviolet; this is signalled by the logarithmic divergences we observed in soft breaking parameters

2. Related to the first point, the soft breakings are all independent parameters.

3. There is no explanation of the large hierarchy.

The gauge mediated models we have introduced to this point represent a significant improvement in that the number of low energy parameters is greatly reduced, in a manner consistent with a broad array of experimental constraints. From the point of view of what we might realistically hope to see at the LHC, this is perhaps enough. but we have not provided any explanation for the parameters $x$ and $F$ which determine the low energy spectrum. In the next section, we will provide examples of weakly coupled, microscopic models in which these parameters are calculable. This could be particularly exciting if some compelling model or dynamical mechanism lead to specific predictions for low energy phenomena, or if some more direct relic of this dynamics might be observable at lower energies. It may also be of interest for trying to connect low energy supersymmetry to some underlying structure (say string theory).

These models will still not address the question of the origin of the hierarchy; we will take up that question when we discuss dynamical supersymmetry breaking in section 9. One of the original motivations for considering supersymmetry as a solution of the hierarchy problem is its susceptibility to dynamical breaking[15]. As we will explain, in (virtually) all situations where supersymmetry is unbroken at tree level, it is unbroken to all orders of perturbation theory. But non-perturbative effects can break the symmetry. So one can envisage that the scale of supersymmetry breaking is given by a formula along the lines:

$$
m_{\text {susy }}=M_{\text {gut }} e^{-\frac{8 \pi^{2}}{c g^{2}\left(M_{\text {gut }}\right)}}
$$

where $g$ is some gauge coupling and $c$ is an order one constant. We will see examples of this sort of phenomenon in section 9. The relevant dynamical effects can be weak coupling, semiclassical phenomena (instantons), or strong coupling dynamics. But we will start by considering models where supersymmetry is broken at weak coupling, already at tree level.

\section{Weakly Coupled Models of Supersymmetry Breaking}

Before writing down models, it is worth considering some general issues. We will focus here on models of global supersymmetry, i.e. we will ignore gravity. Then the condition for supersymmetry breaking is that one not be able to solve the equations:

$$
\frac{\partial W}{\partial \phi_{i}} \neq 0 \forall i \text {. }
$$


These are holomorphic equations, i.e. they depend on the $\phi_{i}$ 's, and not there complex conjugates. It is natural to ask: for such equations, how is it possible that one might not be able to find solutions? Nelson and Seiberg first posed this question in a sharp way, and provided an answer[16]. In order that supersymmetry be broken, it is necessary that some of the equations be inconsistent. If the superpotential is generic, i.e. if one writes down all possible terms, and doesn't insist on special relationships among parameters, this does not happen. If there are $N$ fields, one simply obtains $N$ independent complex equations for $N$ unknowns, and these invariably have solutions. But if the theory possesses an $R$ symmetry, then it is possible to obtain inconsistent equations. Such equations can arise if one has two fields, for example, $X_{1}$ and $X_{2}$, which appear only linearly in the superpotential, i.e.

$$
W=X_{1} f_{1}(\phi)+X_{2} f_{2}(\phi)
$$

where $\phi$ denotes another field. Then unless the zeros of $f_{1}$ coincide with those of $f_{2}$, the equations

$$
\frac{\partial W}{\partial X_{1}}=0 \text { and } \frac{\partial W}{\partial X_{2}}=0
$$

are incompatible.

Without a symmetry, though, there is no reason why there shouldn't be terms in the superpotential involving $X_{1}^{2}, X_{1} X_{2}$, etc. But an $R$ symmetry can account for a structure like that above. This is, loosely speaking, the content of the theorem of Nelson and Seiberg, that $R$ symmetries are required, for models with generic superpotentials, in order to obtain supersymmetry breaking[16]. Recall that under an $R$ symmetry, the superpotential carries $R$ charge two. Then if one has some number of fields, $X_{I}$, with $R$ charge two, and the same number with charge $0, \phi_{i}$, one obtains exactly the structure we have described above.

Note that there are other possibilities. For example, if $I=1, \ldots, N ; i=1, \ldots, n$, then if $n \geq N$, one can, generically, satisfy the equations $\partial W / \partial X_{I}=0$; the $\partial W / \partial \phi_{i}$ equations are satisfied by simply setting the $X$ 's to zero. In general, one has an $n-N$ dimensional space of continuous solutions to these equations; this "moduli space" survives quantum corrections, at least in perturbtion theory, due to supersymmetry (we will discuss this further shortly). If $N>n$, supersymmetry is generically broken, as there are more equations (for the different $I$ 's) than unknowns $\left(\phi_{i}\right.$ 's). Classically, there will be an $N-n$ dimensional moduli space of vacua. Quantum mechanically, this degeneracy will be lifted; these flat directions are thus referred to as "pseudomoduli spaces", and the corresponding fields as pseudomoduli.

\subsection{The O'Raifeartaigh Models}

Models with only chiral fields which implement these ideas are known as O'Raifeartaigh Models. The simplest such model has three fields, $X_{1}, X_{2}, \phi$, and superpotential:

$$
W=\lambda X_{1}\left(\phi^{2}-\mu^{2}\right)+m X_{2} \phi .
$$

This models possess an $R$ symmetry under which $X_{i}$ have $R$ charge 2 ; it is the most general model consistent with symmetries if there is also a discrete symmetry under which both $\phi$ and 
$X_{2}$ are odd, while $X_{1}$ is even. In this model, one has more $X$ type fields than $\phi$ type fields $(N>n)$, so supersymmetry is broken. The equations

$$
\frac{\partial W}{\partial X_{1}}=0 \quad \frac{\partial W}{\partial X_{2}}=0
$$

are incompatible. The equation

$$
\frac{\partial W}{\partial \phi}=2 \lambda X_{1}+m X_{2}=0
$$

just gives a condition on the $X_{i}$, which defines the moduli space.

To determine the vacuum value of $\phi$, we need to minimize the potential

$$
\begin{aligned}
V_{\phi} & =\left|\frac{\partial W}{\partial X_{1}}\right|^{2}+\left|\frac{\partial W}{\partial X_{2}}\right|^{2} \\
& =|\lambda|^{2}\left|\phi^{2}-\mu^{2}\right|^{2}+m^{2}|A|^{2} .
\end{aligned}
$$

If $m^{2}>\lambda^{2} \mu^{2}$, than $\phi=0$ at the minimum, and

$$
\left\langle V_{\phi}\right\rangle=\langle V\rangle=\left|\lambda^{2} \mu^{4}\right|
$$

The order parameter for supersymmetry breaking is

$$
F_{X_{1}}=-\lambda \mu^{2} \quad\left(F_{X_{2}}=0\right) .
$$

$X_{1}$ is undetermined; $X_{2}=0$; this is consistent with the condition for the pseudomoduli of eqn. 43. If $m^{2}<\lambda^{2} \mu^{2}$, one can readily find the (non-zero) expectation value of $\phi$.

The spectrum reflects the breaking of the supersymmetry. In the vacua with $\phi=0$, there is a massless chiral multiplet, $X_{1}$, and a multiplet with the following features:

1. If $X_{1}=0$, there is a Dirac fermion (built from the fermionic components of $\phi$ and $X_{2}$ ), with mass $m$.

2. The complex scalars, $\phi$ and $X_{2}$ (using the same notation here for superfields and their scalar components) have a potential whose quadratic terms are of the form:

$$
\begin{aligned}
V_{\text {scalars }}= & \left|m^{2}\right|\left(|\phi|^{2}+\left|X_{2}\right|^{2}\right) \\
& +\lambda F_{X_{1}}\left(\phi^{2}+\phi^{* 2}\right)
\end{aligned}
$$

so the scalars $X_{2}$ are degenerate with the fermions, but the real and imaginary parts of $\phi^{2}$ are split by $\lambda F_{X_{1}}$. Note that the "supertrace",

$$
\sum(-1)^{F} m_{\alpha}^{2}=0
$$

Here $(-1)^{F}$ is 1 for bosons, -1 for fermions. This is a general result, which is easy to prove, for the spectrum of renormalizable theories at tree level. 
Exercise: By writing the fermion mass matrix for a general theory, and the boson mass matrix, verify the vanishing of the supertrace. The proof can be found in [5], pp. 158-160.

For non-zero $X_{1}$, these basic features still hold. The spectrum is particularly simple if $X_{1}$ is large; then the spectrum is approximately supersymmetric, with $\phi$ possessing mass $\lambda X_{2}$, and real and imaginary parts split, in mass-squared, by $\lambda F_{X_{1}}$. The $X_{2}$ fields have mass of order $m^{2} / \lambda X_{1}$, and with small splittings.

Exercise: Work out the spectrum of the model in the more general vacua for which $X_{1} \neq 0$.

\subsection{The Potential on the Pseudomoduli Space}

The lifting of the vacuum degeneracy in this model can be understood very simply. Classically, the potential is zero. Quantum mechanically, the vacuum energy receives corrections from the zero point fluctuations of the bosons, and the Dirac sea of the fermions. The masses of the fields, as we have just seen, depend of the value of the pseudmodulus, $X_{1}$. As a result, the quantum mechanical contribution to the vacuum energy is a function of $X_{1}$, i.e. there is a potential for $X_{1}$. We can write these two contributions to $V\left(X_{1}\right)$ as:

$$
V\left(X_{1}\right)=\sum(-1)^{F} \int \frac{d^{3} k}{(2 \pi)^{3}} \frac{1}{2} \sqrt{k^{2}+m^{2}\left(X_{1}\right)} .
$$

The individual terms in this expression are divergent, but, due to supersymmetry, there are significant cancelations (these expressions, needless to say, are quite ill defined; the statement that there are cancelations presupposes a regulator which preserves the symmetry). The most severe divergence is the mass-independent, quartic divergence, familiar from introductory field theory texts. This divergence has opposite sign for fermions and bosons, and so cancels due to the equal number of fermionic and bosonic states. The subleading, quadratic divergence is:

$$
\sum(-1)^{F} \int \frac{d^{3} k}{(2 \pi)^{3}} \frac{1}{4} \frac{m^{2}}{k}
$$

which cancels due to the supertrace theorem. Finally, there is a logarithmic divergence, which survives:

$$
-\sum(-1)^{F} \int \frac{d^{3} k}{(2 \pi)^{3}} \frac{1}{8} \frac{m^{4}}{k^{3}}
$$

The result is quadratic in $F_{X_{1}}$; this divergence represents a renormalization of the $X_{1}$ kinetic term, $\int d^{4} \theta X_{1}^{\dagger} X_{1}$.

We can quickly do the calculation for large $X_{1}$. Then the masses are all approximately equal to $\left|\lambda X_{1}\right|$, so we can approximate the $\log$ by $\log \left|X_{1}\right| ;(-1)^{F} m^{4}=4\left|\lambda^{2} F_{x}^{2}\right|$. So

$$
V\left(X_{1}\right) \approx 4 \frac{\left|\lambda^{2} F_{X_{1}}^{2}\right|}{4 \pi^{2}} \log \left(\left|\lambda^{2} X_{1}\right|\right)
$$


This potential grows for large $X_{1}$. For smaller $X_{1}$, it is straightforward to check that the minimum of the potential is at the origin. This means, in particular, that the $R$ symmetry is not spontaneously broken. When we come to model-building, this will be important.

Shih has shown that this is completely general; in models in which all fields have $R$ charge 0 or 2 , the $R$ symmetry is always unbroken[17]. Models with different $R$ charge assignments can break the symmetry. The simplest such model has fields with $R$ charge $2,1,-1$ and 3 , which we will denote by $X, \phi_{1}, \phi_{-1}, \phi_{3}$ :

$$
W=-\mu^{2} X+\lambda X \phi_{-1} \phi_{1}+m_{1} \phi_{1} \phi_{1}+m_{2} \phi_{-1} \phi_{3} .
$$

This model, for a range of parameters, has an $R$-symmetry breaking minimum.

\subsubsection{Complete Models}

With our results above, we can now demonstrate the existence of microscopic models of models of gauge mediation. We can simply couple the field $X$ of the model of eqn. 53 to messenger fields, as in minimal gauge mediation. In this model, as it stands, $\mathrm{CP}$ is unbroken and the $R$ symmetry is broken, so we can obtain gaugino masses without large phases. However, it is necessary to introduce additional fields and couplings in order to generate a $\mu$ term, and this can lead to additional phases and $\mathrm{CP}$ violation.

\subsection{Metastable Supersymmetry Breaking}

It is unlikely that any fundamental theory exhibits continuous symmetries; it is a theorem in string theory that there are no global symmetries[18]. At best, then, the $R$ symmetry of models like the O'Raifeartaigh models will be approximate. In the case of the model of eqn. 41, for example, the continuous $R$ symmetry might be a consequence of a discrete $R$ symmetry under which

$$
\begin{aligned}
X_{1} \rightarrow e^{\frac{2 \pi i}{N}} X_{1} \quad X_{2} \rightarrow e^{\frac{2 \pi i}{N}} X_{2} \\
\theta \rightarrow e^{\frac{\pi i}{N}} \theta \quad W \rightarrow e^{\frac{2 \pi i}{N}} W
\end{aligned}
$$

and $\phi$ is invariant (this is, again, an $R$ symmetry because the superpotential transforms, and so do the supercharges). Along with the $X_{2} \rightarrow-X_{2}, \phi \rightarrow-\phi$ symmetry, this accounts for the structure of the lagrangian, at the renormalizable level. But couplings like $\frac{1}{M^{N-2}} X_{1}^{N+1}$ are allowed by the symmetry. As a result, the equation

$$
\frac{\partial W}{\partial X_{1}}=0
$$

has a solution, with $X_{1}$ large $\left(X_{1}^{N} \sim M^{N-2} \mu^{2}\right)$.

What of the non-supersymmetric vacuum? Classically, the high dimension coupling of eqn. 55 destabilizes this state; more precisely it gives rise to a classical potential on the original 
moduli space, with a minimum at the supersymmetric point. However, the quantum mechanical corrections we have evaluated above render this state a local minimum of the potential. The nonsupersymmetric vacuum is metastable; it's lifetime is exponentially long, where the exponent scales as a power of $M / \mu$.

Indeed, the Nelson-Seiberg theorem, which requires a continuous R symmetry, and the fact that we don't expect continuous global symmetries in sensible fundamental theories, suggests that this behavior should be generic. We will consider this issue again when we discuss models of dynamical supersymmetry breaking.

\section{Dynamical Supersymmetry Breaking}

So far, the parameters $\mu$ and $m_{i}$ in our various models were introduced by hand. If they are to be connected to the hierarchy problem, they must be hierarchically small. Supersymmetry is prone to generating small numbers[15]. Most dramatically, if supersymmetry is unbroken at tree level, it is typically unbroken to all orders of perturbation theory. This follows from a set of non-renormalization theorems. Originally, these theorems were understood by studying the structure of perturbation theory in supersymmetric theories[19]; Seiberg explained how to understand these theorems in a much more conceptual way. This understanding indicates that the theorems (as originally speculated by Witten and demonstrated by [20]) do not extend beyond perturbation theory, opening the possibility of generating a large hierarchy, of order $e^{-c / g^{2}}$.

\subsection{Non-Renormalization Theorems}

Seiberg[21], in a program that has had far reaching implications, realized that these theorems could be understood far more simply. Moreover, Seiberg's proof indicates clearly when nonperturbative effects can violate the theorems. His ingenious suggestion was to consider the couplings in the superpotential, and the gauge couplings, as expectation values of chiral fields. These fields must appear holomorphically in the superpotential and gauge coupling functions, and this greatly restricts the coupling dependence of these quantities.

To illustrate, consider a simple Wess-Zumino model:

$$
W=\frac{1}{2} m \phi^{2}+\frac{1}{3} \lambda \phi^{3} .
$$

For $\lambda=0$, this model possesses an $\mathrm{R}$ symmetry, under which $\phi$ has $R$ charge 1 . So we can think of $\lambda$ as a chiral field with $R$ charge -1 . Since the superpotential is holomorphic, the only allowed terms, polynomial in the $\phi$ 's, have the form

$$
\Delta W=\sum_{n} \lambda^{n} \phi^{n+3} .
$$

This is precisely the $\lambda$ dependence of tree diagrams with $n+3$ external legs; we have predicted that there are no loop corrections to the superpotential. 
Note that there is no corresponding argument for the Kahler potential, and it is easy to check that the Kahler potential is already renormalized at one loop. As a result, physical masses and couplings are corrected in this model. But the non-renormalization theorems have, as we will see, profound significance.

For gauge theories, the results are in many ways even more dramatic and surprising. Again, the coupling can be represented as a complex field:

$$
\mathcal{L}=-\frac{1}{4} \int d^{2} \theta \tau W_{\alpha}^{2}
$$

where $\tau=\frac{1}{g^{2}}+i \frac{\theta}{8 \pi^{2}}$ and $\theta$ is the usual CP-violating parameter of the gauge theory. Perturbation theory is insensitive to $\theta$ (for an introduction to the $\mathrm{CP}$ violating parameter, $\theta$, see, for example, [5], chapter 5) so, order by order, the Wilsonian effective action is

$$
\tau \rightarrow \tau+i \epsilon
$$

Apart from $\mathcal{L}$, the only combination of $\tau$ and $W_{\alpha}^{2}$ which is invariant under the shift symmetry is $\int d^{2} \theta W_{\alpha}^{2}$, which is precisely the structure of the one loop correction. So we seem to establish that there is at most a one loop correction to the gauge coupling, and that there are no loop corrections to the superpotential. The gauge coupling result is puzzling, since it is well-known that there is a two loop correction to the beta-function in supersymmetric gauge theories. This issue has a resolution, due to Shifman and Vainshtein[31]; I do not have time to explain it fully here (see, for example, [5], pp. 501-503), but heuristically, the issue can be understood by realizing that the cutoff of the theory, itself, is not, in general, a holomorphic function of the coupling (equivalently, the question is one of renormalization scheme).

It is well-known that the shift symmetry of perturbation theory is anomalous, and is broken beyond perturbation theory (for an introduction, see [5], chapter 5). In an $\mathrm{SU}(\mathrm{N})$ gauge theory, for example, instantons generate an expectation value for:

$$
\left\langle(\lambda \lambda)^{N}\right\rangle \propto e^{-\frac{8 \pi^{2}}{g^{2}}+i \theta}=e^{-8 \pi^{2} \tau} .
$$

This expectation value leaves over a discrete $Z_{N}$ symmetry. It has long been believed - and using the sorts of holomorphy arguments developed by Seiberg, shown - that gluinos condense in this theory:

$$
\left\langle W_{\alpha}^{2}\right\rangle=\langle\lambda \lambda\rangle=\Lambda^{3} e^{i \theta / N} \propto e^{-3 \tau / b_{0}} .
$$

One can think of this as a constant superpotential, so it represents a breakdown of the non-renormalization theorems. By itself, this is not so interesting. In global supersymmetry, physics is not sensitive to a constant $W$ (though in local supersymmetry, if one started in flat space, one would now have a theory with unbroken supersymmetry in anti-De Sitter space). But now couple the gauge theory to a singlet, $S$, with no other couplings:

$$
\mathcal{L}=\left(\tau+\frac{S}{M}\right) W_{\alpha}^{2}
$$


Then

$$
W_{e f f}(S) \propto e^{\frac{-\tau}{3 b_{0}}} e^{-\frac{1}{3 b_{0}} \frac{S}{M}}
$$

So a classical moduli space $(S)$, has been lifted; we have breakdown of the non-renormalization theorems and dynamical supersymmetry breaking through non-perturbative effects.

\subsection{Retrofitting of the O'Raifeartaigh Model}

Of course, this is not quite what we are looking for. The potential for $S$ simply falls to zero at large $S$; if it has a stationary point, it lies in a region where the effective coupling is large and we cannot calculate. Moreover, such a stationary point will not be metastable, as there is no small parameter to suppress the decay rate.

But we can use our experience with O'Raifeartaigh models to build a theory with metastable supersymmetry breaking. Introduce singlet fields, $X, Y$ and $\phi$, and take for the superpotential and gauge couplings of the model[22]:

$$
\mathcal{L}=\frac{X}{M} W_{\alpha}^{2}+X \phi^{2}+m Y \phi
$$

At energies below the scale of the strong gauge group, $\Lambda$, we can integrate out the gauge interactions leaving the effective superpotential:

$$
W(X, A, Y)=X \phi^{2}+\Lambda^{3} e^{-\frac{1}{N} \frac{X}{M}}+m Y \phi .
$$

Near $X=0$, this is like the OR model, and one loop corrections will again generate a local minimum of the potential. But at very large $X$ ( $X$ comparable to $M)$, the potential falls away to zero. A model of this type is said to be retrofitted.

We would like to account for the mass scale $m$ dynamically as well; we might wish, for example, that $\mu_{\text {eff }}^{2}=\frac{\Lambda^{3}}{M} \sim m^{2}$. We will discuss how this can naturally be achieved in the next subsection, where we discuss the role of symmetries in such models.

\subsubsection{Symmetries of the Retrofitted Model}

In the case of metastable OR models, we saw that discrete symmetries could account for the approximate $R$ symmetry of the model. In the present case, discrete symmetries can also play this role, though they look somewhat different than those we encountered earlier, since $W_{\alpha}^{2}$ necessarily transforms like the superpotential. As one possibility, consider:

$$
W_{\alpha} \rightarrow e^{\frac{2 \pi i}{N}} W_{\alpha} X \rightarrow X \phi \rightarrow e^{\frac{2 \pi i}{N}} \phi Y \rightarrow e^{\frac{2 \pi i}{N}} Y
$$

It is necessary to forbid $Y^{2}$ and $Y^{2} X$; this can be achieved by suitable additional ordinary symmetries (e.g. a $Z_{4}, Y \rightarrow i Y, \phi \rightarrow-i \phi$ ). The continuous $R$ symmetry of the low energy theory is then a consequence of these discrete symmetries, and the restriction to low order dimension terms in the effective action. 
We can account for the scale $m$ of the low energy theory dynamically through a structure such as[23]:

$$
\Delta \mathcal{L}=\int d^{2} \theta\left(\frac{X W_{\alpha}^{2} W_{\beta}^{2}}{M^{4}}+X \phi^{2}+\frac{W_{\alpha}^{2}}{M^{2}} Y \phi\right) .
$$

This model achieves $m^{2} \sim \mu^{2}$. Starting with models such as this, we can again couple to messengers and build models of gauge mediated supersymmetry breaking.

Exercise: Show that the superpotential of eqn. 67 is the most general consistent with symmetries, through cubic order in the fields.

\section{Supersymmetry Dynamics}

The retrofitted construction is, in some sense, almost too easy. One could conceive of structures such as this emerging from a more fundamental theory, but it is worth enlarging our perspective on dynamical supersymmetry breaking.

\section{$10.1 \quad$ Supersymmetric QCD}

Let's step back and think more about supersymmetric dynamics. We first consider Supersymmetric $Q C D$ with $N_{f}$ flavors, which we will define to be a supersymmetric theory with gauge group $S U(N)$ and $N_{f}$ quarks in the $N$ and $N_{f}$ in the $\bar{N}$ representations, $Q_{f}, \bar{Q}_{\bar{f}}$. Consider, first, the theory with massless quarks. The model has a global symmetry $S U\left(N_{f}\right) \times S U\left(N_{f}\right) \times$ $U(1)_{B} \times U(1)_{R}$. Here we are listing only symmetries free of anomalies. $Q$ and $\bar{Q}$ transform as

$$
\begin{aligned}
& Q:\left(N_{f}, 1, \frac{1}{N}, \frac{N_{f}-N}{N_{f}}\right) \\
& \bar{Q}:\left(1, N_{f},-\frac{1}{N}, \frac{N_{f}-N}{N_{f}}\right) .
\end{aligned}
$$

Let's check the cancelation of anomalies. We are concerned about triangle diagrams with the symmetry current at one vertex, and $S U(N)$ gauge bosons at the other two vertices. For the $S U\left(N_{f}\right)$ symmetry, the absence of anomalies is automatic (resulting from the tracelessness of the $S U\left(N_{f}\right)$ generators; for $U(1)_{B}$ it follows immediately from the opposite baryon numbers of $Q$ and $\bar{Q}$. For the $R$ symmetry, note that the $R$ charge of the gluino is +1 , so the gluino makes a contribution to the anomaly proportional to $N$ (the Casimir of the adjoint representation); the $R$ charges of the (fermionic) quarks and antiquarks, $\psi_{Q}$ and $\psi_{\bar{Q}}$ are $\frac{N_{f}-N}{N_{f}}-1=-\frac{N}{N_{f}}$. So, as the Casimir of the fundamental is $1 / 2$, and there are $2 N_{f}$ fields of the same $R$ charge contributing to the anomaly, we obtain cancelation.

It is important to understand the structure of the massless theory. Classically, there is a large moduli space of SUSY vacua. The potential arises simply from the $D^{2}$ terms of the gauge 
fields; it is enough to ensure that these vanish. Up to gauge and flavor transformations, for $N_{f}<N$,

$$
Q=\left(\begin{array}{cccccc}
v_{1} & 0 & 0 & \ldots & 0 & \ldots \\
0 & v_{2} & 0 & \ldots & 0 & \ldots \\
& & & & & \ldots \\
0 & 0 & 0 & \ldots & v_{N_{f}} & \ldots
\end{array}\right)
$$

and $Q=\bar{Q}$.

Exercise: Verify the statement above. This can be done in a variety of ways. First, note that the vanishing of the $D$ terms with $Q, \bar{Q}$ as above is automatic. It follows from the "messenger parity" symmetry we have described above, under which the $D$ terms are odd. It also follows from the explicit form of the $D$ terms. These can be written as $S U(N)$ matrices (see, for example, [5], chapter 13)

$$
D_{j}^{i}=Q^{* i} Q_{j}-\bar{Q}^{i} \bar{Q}_{j}^{*}-\text { Trace }
$$

One can show this is the most general solution by first using the gauge and flavor symmetries to bring $Q$ to the form, above, and then arguing that, in virtue of eqn. 70, $\bar{Q}$ is identical, up to flavor transformations.

In these directions, the gauge symmetry is broken to $S U\left(N-N_{F}\right)$. There are $N^{2}-(N-$ $\left.N_{f}\right)^{2}=2 N N_{f}-N_{f}^{2}$ broken generators. Each broken generator "eats" one chiral field. There are also a set of broken flavor symmetries. For example, if the $v$ 's are all equal, the unbroken flavor symmetry is $U(1) \times S U\left(N_{f}\right)$, so there are $N_{f}^{2}-1+1$ Goldstone fields; these also arise from the chiral fields; in this way we have accounted for all of the $Q, \bar{Q}$ fields. We can understand this counting another way, by constructing $N_{f}^{2}$ gauge invariant "meson" fields:

$$
M_{f, \bar{f}}=\bar{Q}_{\bar{f}} Q_{f} .
$$

Perturbatively, these directions remain flat. Non-perturbatively, there is a unique superpotential which one can write which is consistent with the symmetries:

$$
W=\frac{\Lambda^{\frac{3 N+N_{f}}{N-N_{f}}}}{\operatorname{det}\left(M_{f, \bar{f}}\right)} .
$$

In the case $N_{f}=N-1$, one can compute the superpotential in a straightforward semiclassical analysis. This is described in [5], section 13.6.

Exercise: Verify that the superpotential of equation 72 respects the $S U\left(N_{f}\right)$ and non-anomalous $U(1)_{R}$ symmetries. Go one step further. Introduce the background field $\tau$, and assign it a transformation law, $\tau \rightarrow \tau+i C \alpha$, choosing the constant $C$ such that the $\tau$ transformation cancels the anomaly in the symmetry under which $Q, \bar{Q}$ have $\mathrm{R}$ charge zero. Recalling the dependence of $\Lambda$ on $\tau$, verify that 72 respects this symmetry as well. 
As for our simplest retrofitted model, the potential associated with $W$, while it lifts the moduli, does not yield a stationary point of the potential in any regime where the coupling is weak. Again, it is conceivable that there are stationary points in the strong coupling region, but such would-be states will have unsuppressed decays, i.e. they are not really states at all.

But there are several interesting questions we can study using these results. First, we can derive the fact of gaugino condensation. We'll exhibit the result for $S U(2)$. Start with $N=2$, with one flavor. Then in the general flat direction, the gauge symmetry is completely broken, and there is a single light meson, $\Phi=\bar{Q} Q$; the superpotential can be systematically computed in the semiclassical approximation. If we include a small mass for $\Phi$, we have a superpotential

$$
W=m \Phi+\frac{\Lambda^{5}}{\Phi}
$$

This superpotential has supersymmetric stationary points for

$$
\Phi=e^{\frac{2 \pi i}{N}}\left(\frac{\Lambda^{5}}{m}\right)^{1 / 2}\langle W\rangle=e^{\frac{2 \pi i}{N}} \Lambda^{5 / 3} m^{1 / 2}
$$

where we have written the phase to point to the generalization to the case of $S U(N)$ with $N-1$ flavors. Now Seiberg points out that $\langle W\rangle$ is a holomorphic function of $m$ and $\Lambda$, and it must transform properly under the symmetries (with $m$ and $\Lambda$ treated as background fields). As a result, this expression is valid for all $m$, and in particular for $m$ large, so that one is studying, at low energies, a pure $S U(2)$ gauge theory. In that theory, $\langle W\rangle=\langle\lambda \lambda\rangle$.

Exercise: Verify that the dependence on $m$ and $\Lambda$ of $\langle W\rangle$ is uniquely determined by symmetries (see [5], p. 208).

\subsection{Varieties of Dynamical Supersymmetry Breaking}

Modifying slightly the theories we have designated as Supersymmetric QCD, we will be able to uncover two types of supersymmetry breaking. One is stable, in the sense that, at the renormalizable level (more generally, up to operators of some fixed dimension), there are only supersymmetry-breaking ground states (related to one another by symmetries); the second is metastable[24], much like the theories we have encountered in the weakly coupled and retrofitted cases, where, even at the renormalizable level, there are additional supersymmetric states, separated by a large barrier or a sizable distance in field space.

\subsubsection{Stable Supersymmetry Breaking - the $3-2$ Model}

Models with stable supersymmetry breaking are rare. They are generally characterized by two features:

1. Classically, their potentials have no flat directions (there is not a moduli space of vacua). 
2. They exhibit global symmetries, which are spontaneously broken in the ground state.

If the first condition is not satisfied, then, as we have seen, there are typically regions in the moduli space where the potential tends to zero, corresponding to (at least) asymptotic restoration of supersymmetry. If the second condition is satisfied, there is some number of Goldstone particles. In general, as in the example of massless QCD, these particles each lie in a different chiral multiplet. The other scalar in the multiplet, like the Goldstone, will have no potential; it is a flat direction, contradicting the first assumption above. There is a potential loophole in this argument: it is logically possible that both fields in the multiplet are Goldstone particles. Typically, however, this does not occur.

The simplest example of such a theory, in which it is possible to do systematic calculations, is known as the $3-2$ model because the gauge group is $S U(3) \times S U(2)$. Its particle content is like that of a single generation of the Standard Model, minus the singlet:

$$
Q:(3,2) \bar{U}:(\overline{3}, 1) \bar{D}:(\overline{3}, 1) L=(1,2) .
$$

There is a unique superpotential allowed by the symmetries, up to field redefinitions:

$$
W=\lambda Q L \bar{U}
$$

Without the superpotential, and assuming that the $S U(2)$ coupling is much smaller than the $S U(3)$ coupling, this is supersymmetric QCD with $N=3, N_{f}=2$. The theory has a set of flat directions, and generates a non-perturbative superpotential. It is easy to see, however, that the classical superpotential of eqn. 76 already lifts all of the flat directions. Moreover, the theory possesses a non-anomalous $R$ symmetry.

Exercise: First show that, without loss of generality, you can take, for the flat direction of the $D$-term:

$$
\begin{gathered}
Q=\left(\begin{array}{lll}
a & 0 & 0 \\
0 & b & 0
\end{array}\right) \quad L=\left(\begin{array}{c}
e^{i \phi} \sqrt{|a|^{2}-|b|^{2}} \\
0
\end{array}\right) \\
\bar{U}=\left(\begin{array}{lll}
a & 0 & 0
\end{array}\right) \quad \bar{D}=\left(\begin{array}{lll}
0 & b & 0
\end{array}\right) .
\end{gathered}
$$

Now show that for any choice of $\bar{U}$, one cannot satisfy all of the $\frac{\partial W}{\partial \phi_{i}}$ 's to zero, unless both $a$ and $b$ vanish. Finally, check that the model possesses a non-anomalous $R$ symmetry which is spontaneously broken by a non-vanishing $a$ or $b$.

For small $\lambda$, the effective superpotential is:

$$
W_{e f f}=\frac{\Lambda^{6}}{Q Q \bar{U} \bar{D}}+\lambda Q L \bar{U}
$$

Careful study of the resulting potential exhibits a supersymmetry-breaking minimum.

One can ask: what happens in this model if the $S U(2)$ coupling is much greater than the $S U(3)$ coupling, so that the $S U(2)$ gauge group becomes strong first. In this limit, the 
theory looks like $Q C D$ with $N=2, N_{f}=2$. In this theory, there is no non-perturbative superpotential: there exists an exact moduli space, even quantum mechanically. However, as Seiberg showed, in such theories, the moduli space is modified quantum mechanically. In effect, $Q L$ is non-zero everywhere on the moduli space, generating an $F$ term for $\bar{U}$ through eqn. 76[25] ( for a pedagogical discussion of the 3-2 model, including this issue, see [5], sections 14.1, 16.3.1).

Finally, what if we give up the requirement of renormalizability? Adding higher dimension operators (e.g. $\left.(Q \bar{U} L)^{2}\right)$, with coefficients scaled by a large mass, $M$, we will lose the continuous $R$ symmetry as an exact symmetry, and there will be supersymmetric minima. These minima, however, will be far away, and separated by a large barrier (with barrier height scaled by $M$ ) from the non-supersymmetric minimum near the origin. The metastable state near the origin will be extremely stable.

It is not easy to find theories which satisfy the conditions for stable supersymmetry breaking, and those which exist pose challenges for model building. To illustrate the issues, we can consider a class of models with gauge group $S U(N)$, and an antisymmetric tensor, $A_{i j}$, as well as $N-4$ antifundamentals, $\bar{F}$. The simplest of these models has $N=5$, and a single $\overline{5}$. It is easy to check, using the matrix technique developed above, that there are no flat directions of the $D$ terms. There is a non-anomalous $R$ symmetry; one can give arguments that, in this strongly coupled theory, the symmetry is spontaneously broken[26]. These features extend to the model with general $N$, when we include the most general superpotential

$$
W=\lambda_{a b} \bar{F}_{i}^{a} \bar{F}_{j}^{b} A^{i j}
$$

So we might adopt the following strategy for model building. Take $N_{f}$ (and hence $N$ ) and choose $\lambda$ appropriately so that the model has a large flavor group. For example, for $N=14$, the flavor group can include $S U(5)$; gauge a subgroup of the flavor group, and identify this subgroup with the Standard Model gauge group. The difficulty is that the QCD and other gauge couplings are, in this case, violently non-asymptotically free. Unification is lost; indeed, some enlargement of the Standard Model group is required only a few decades above the susy-breaking scale. Most model building with stable supersymmetry breaking invokes more complicated structures in order to obtain a vev for a field like $X$, which in turn couples to messengers. The constructions are rather baroque[27].

\subsubsection{Metastable Supersymmetry Breaking: The ISS Model}

We have seen that for $N_{f}<N$, there is a potential generated non-perturbatively on the classical moduli space. For $N_{f} \geq N$, this is not the case; there is always an exact moduli space. But the dynamics on this moduli space, particularly in the region of strong coupling, is quite intricate, with a strong dependence on the values of $N$ and $N_{f}[28]$ (for short, pedagogical introductions, see [29] and [5], chapter 16).

One interesting range is $N+1<N_{f}<3 / 2 N$. Here, the theory is dual to a theory with

gauge group $S U\left(N_{f}-N\right)$, with $N_{f}$ flavors of quarks, $q_{f}$ and $\bar{q}_{f}$, and a set of mesons, $M_{f, \bar{f}}$; the 
effective lagrangian of this theory possesses a superpotential:

$$
W=\bar{q} M q .
$$

This duality is not meant as an exact equivalence, but rather a statement about the infrared behaviors of the two theories.

We have seen that massive QCD has $N$ supersymmetric vacua, connected with the breaking of the $Z_{N}$ symmetry of the theory. Long ago, Witten, in fact, proved that there were $N$ such vacua, and as a result, these theories were not viewed as an interesting arena for dynamical supersymmetry breaking[30]. But Intriligator, Shih and Seiberg[24] made the following remarkable observation about theories in this range of $N_{f}$. They considered adding a small mass term for the quarks, $m \bar{Q} Q$ in the ultraviolet theory. Then, in the infrared, "magnetic", theory the superpotential is:

$$
W_{\text {mag }}=\bar{q} M q+\operatorname{Tr} m \Lambda M .
$$

This superpotential does not have supersymmetric minima.

Exercise: Verify that the additional term transforms correctly under the $S U\left(N_{f}\right)$ and $U(1)_{R}$ symmetries of the underlying theory.

Exercise: Show that the $D$ term conditions and $F$ term conditions in the magnetic theory cannot be satisfied simultaneously. One can first bring $q$ to the following form, using gauge and flavor transformations

$$
q_{f}^{i}=v_{i} \delta_{i f} \quad f<i ; \quad \text { zero otherwise }
$$

Then the vanishing of the $D$ terms allows one to bring $\bar{q}$ to the same form, up to flavor transformations. Now it is straightforward to see that the $\partial W / \partial M_{f \bar{f}}$ conditions cannot be satisfied, for $f>N$. This type of breaking is called "rank breaking" by the authors of [24].

At the classical level, the magnetic lagrangian gives rise to moduli; $M_{f \bar{f}}$ is not fixed, for example. Almost as remarkable as the fact that supersymmetry is broken is the fact that one can compute the potential for $M, q \bar{q}$ near the origin, even though the theory is not weakly coupled. The result of this computation is that the minimum of the potential does not break the $R$ symmetry (the expectation value of $M$ vanishes). For model building, this is problematic, at least if supersymmetry is to be broken at a low scale. But this is likely the tip of the iceberg of a large class of strongly coupled models exhibiting metastable supersymmetry breaking. ${ }^{1}$

We should pause and ask: where are the $N$ supersymmetric vacua in the magnetic description? These can be understood in the dual picture. We remarked that in the metastable vacuum, the $R$ symmetry is unbroken, but we expect that the supersymmetric vacua should exhibit broken discrete $R$ symmetries. So consider giving $M$ a large expectation value. Then all of the dual quarks are massive, and the low energy theory is asymptotically free. Gaugino condensation leads to an additional term in the superpotential for $\Phi$, and one finds $N$ supersymmetric vacua.

\footnotetext{
${ }^{1} \mathrm{~A}$ model building program with a low scale ISS-type model has been developed by Banks and collaborators[32].
} 


\section{Conclusions}

Physicists have been considering supersymmetry as a major component of electroweak symmetry breaking for nearly 30 years. The ideas surrounding gauge mediation are nearly as old, and models of dynamical supersymmetry breaking have been known for 25 years. Yet the last three years have seen significant developments. The appreciation that metastable supersymmetry breaking is likely to be an important component of supersymmetry breaking has opened up vast possibilities for model building; with this has come the realization that even for gauge mediation, the possible spectra and phenomenology[33] is much richer than might have been imagined.

As of this writing, the start of physics from the LHC looks to be about one year away. It is possible that we are on the brink of resolving the origin of electroweak symmetry breaking and the hierarchy problem; if so, supersymmetry likely plays some role. But given that the scale of supersymmetry breaking might reasonably be expected to be of order $M_{Z}$, it is troubling that we have seen no direct evidence of supersymmetry already. I have tried to suggest, in these lectures, that while this lack of evidence may well be argue against supersymmetry, it may instead be telling us that underlying supersymmetry breaking is dynamics at some relatively low energy scale, mediated to ordinary fields by gauge interactions.

\section{Acknowledgements}

I am grateful to my collaborators on the projects alluded to here. In recent years, these include Linda Carpenter, Jonathan Feng, Guido Festuccia, John Mason, Nathan Seiberg, Eva Silverstein and Scott Thomas. This work supported in part by the U.S. Department of Energy.

\section{References}

[1] S. Weinberg, Phys. Rev. Lett. 59, 2607 (1987).

[2] G. F. Giudice and R. Rattazzi, Phys. Rept. 322, 419 (1999) [arXiv:hep-ph/9801271].

[3] L. Girardello and M. T. Grisaru, Nucl. Phys. B 194, 65 (1982).

[4] S. Dimopoulos and H. Georgi, Nucl. Phys. B 193, 150 (1981).

[5] M. Dine, Supersymmetry and String Theory: Beyond the Standard Model, Cambridge University Press, Cambridge (2006).

[6] A. Masiero and O. Vives, New J. Phys. 4, 4 (2002).

[7] For a review of the issues, see M. Ciuchini, E. Franco, A. Masiero and L. Silvestrini, Phys. Rev. D 67, 075016 (2003) [Erratum-ibid. D 68, 079901 (2003)] [arXiv:hep-ph/0212397]; for a relatively recent summary of the theoretical and experimental situation, with references, see U. Haisch, arXiv:0706.2056 [hep-ph].

Paper 1 to 1 of 1 
[8] R. Essig and J. F. Fortin, JHEP 0804, 073 (2008) [arXiv:0709.0980 [hep-ph]].

[9] M. Dine, N. Seiberg and S. Thomas, Phys. Rev. D 76, 095004 (2007) [arXiv:0707.0005 [hep-ph]].

[10] G. F. Giudice and R. Rattazzi, Nucl. Phys. B 511, 25 (1998) [arXiv:hep-ph/9706540]; N. Arkani-Hamed, G. F. Giudice, M. A. Luty and R. Rattazzi, Phys. Rev. D 58, 115005 (1998) [arXiv:hep-ph/9803290].

[11] P. Meade, N. Seiberg and D. Shih, arXiv:0801.3278 [hep-ph].

[12] Z. Komargodski and N. Seiberg, arXiv:0812.3900 [hep-ph].

[13] M. Buican, P. Meade, N. Seiberg and D. Shih, arXiv:0812.3668 [hep-ph].

[14] G. F. Giudice, H. D. Kim and R. Rattazzi, Phys. Lett. B 660, 545 (2008) [arXiv:0711.4448 [hep-ph]]; G. R. Dvali, G. F. Giudice and A. Pomarol, Nucl. Phys. B 478, 31 (1996) [arXiv:hep-ph/9603238].

[15] E. Witten, Nucl. Phys. B 188, 513 (1981).

[16] A. E. Nelson and N. Seiberg, Nucl. Phys. B 416, 46 (1994) [arXiv:hep-ph/9309299].

[17] D. Shih, JHEP 0802, 091 (2008) [arXiv:hep-th/0703196].

[18] T. Banks and L. J. Dixon, Nucl. Phys. B 307, 93 (1988).

[19] M. T. Grisaru, W. Siegel and M. Rocek, Nucl. Phys. B 159, 429 (1979).

[20] I. Affleck, M. Dine and N. Seiberg, Phys. Rev. Lett. 51, 1026 (1983).

[21] N. Seiberg, Phys. Lett. B 318, 469 (1993) [arXiv:hep-ph/9309335].

[22] M. Dine, J. L. Feng and E. Silverstein, Phys. Rev. D 74, 095012 (2006) [arXiv:hepth/0608159].

[23] M. Dine and J. D. Mason, arXiv:0712.1355 [hep-ph].

[24] K. A. Intriligator, N. Seiberg and D. Shih, JHEP 0604, 021 (2006) [arXiv:hep-th/0602239].

[25] K. A. Intriligator and S. D. Thomas, arXiv:hep-th/9608046.

[26] I. Affleck, M. Dine and N. Seiberg, Phys. Lett. B 137, 187 (1984).

[27] M. Dine and A. E. Nelson, Phys. Rev. D 48, 1277 (1993) [arXiv:hep-ph/9303230]; M. Dine, A. E. Nelson and Y. Shirman, Phys. Rev. D 51, 1362 (1995) [arXiv:hep-ph/9408384]; M. Dine, A. E. Nelson, Y. Nir and Y. Shirman, Phys. Rev. D 53, 2658 (1996) [arXiv:hep$\mathrm{ph} / 9507378]$.

[28] N. Seiberg, Nucl. Phys. B 435, 129 (1995) [arXiv:hep-th/9411149]; N. Seiberg, Phys. Rev. D 49, 6857 (1994) [arXiv:hep-th/9402044].

[29] M. E. Peskin, arXiv:hep-th/9702094. 
[30] E. Witten, Nucl. Phys. B 202, 253 (1982).

[31] M. A. Shifman, A. I. Vainshtein and V. I. Zakharov, Phys. Lett. B 166, 334 (1986);

M. A. Shifman and A. I. Vainshtein, Nucl. Phys. B 359, 571 (1991).

[32] T. Banks, arXiv:hep-ph/0606313.

[33] L. M. Carpenter, arXiv:0812.2051 [hep-ph]. 